\title{
Recurrent Falls in People with Parkinson's Disease without Cognitive Impairment: Focusing on Modifiable Risk Factors
}

\author{
Lorena R. S. Almeida, ${ }^{1,2}$ Guilherme T. Valença, ${ }^{1,2}$ Nádja N. Negreiros, \\ Elen B. Pinto, ${ }^{2}$ and Jamary Oliveira-Filho ${ }^{2}$ \\ ${ }^{1}$ Movement Disorders Clinic, Bahia State Health Attention Center for the Elderly, Avenida ACM, s/n, \\ Ed. José Maria de Magalhães Netto, Iguatemi, 41820-000 Salvador, BA, Brazil \\ ${ }^{2}$ Post-Graduate Program in Health Sciences, Federal University of Bahia, Largo do Terreiro de Jesus, $s / n$, \\ Centro Histórico, 40025-010 Salvador, BA, Brazil
}

Correspondence should be addressed to Lorena R. S. Almeida; lorenasantos@gmail.com

Received 6 July 2014; Revised 21 October 2014; Accepted 4 November 2014; Published 23 November 2014

Academic Editor: Francisco Grandas

Copyright (C) 2014 Lorena R. S. Almeida et al. This is an open access article distributed under the Creative Commons Attribution License, which permits unrestricted use, distribution, and reproduction in any medium, provided the original work is properly cited.

Falls can be considered a disabling feature in Parkinson's disease. We aimed to identify risk factors for falling, testing simultaneously the ability of disease-specific and balance-related measures. We evaluated 171 patients, collecting demographic and clinical data, including standardized assessments with the Unified Parkinson's Disease Rating Scale (UPDRS), activities of daily living (ADL) and motor sections, modified Hoehn and Yahr Scale, Schwab and England, eight-item Parkinson's Disease Questionnaire, Activitiesspecific Balance Confidence Scale, Falls Efficacy Scale-International (FES-I), Berg Balance Scale, Dynamic Gait Index, Functional Reach, and Timed Up and Go. ROC curves were constructed to determine the cutoff scores for all measures. Variables with $P<$ 0.1 entered a logistic regression model. The prevalence of recurrent falls was 30\% (95\% CI 24\%-38\%). In multivariate analysis, independent risk factors for recurrent falls were $(P<0.05)$ levodopa equivalent dose $(\mathrm{OR}=1.283$ per $100 \mathrm{mg}$ increase; $95 \% \mathrm{CI}=$ 1.092-1.507), UPDRS-ADL > 16 points $(\mathrm{OR}=10.0 ; 95 \% \mathrm{CI}=3.6-28.3)$, FES-I $>30$ points $(\mathrm{OR}=6.0 ; 95 \% \mathrm{CI}=1.6-22.6)$, and Berg $\leq 48$ points $(\mathrm{OR}=3.9 ; 95 \% \mathrm{CI}=1.2-12.7)$.We encourage the utilization of these modifiable risk factors in the screening of fall risk.

\section{Introduction}

Parkinson's disease (PD) is a common neurodegenerative disorder in older adults and postural instability is well known to occur in these individuals, increasing the risk for falling [14]. Falls are one of the most disabling features of PD and the incidence of falls is high, with reported rates varying from 35 to $68 \%$ for one or more falls [5-12] and from 24 to $43 \%$ for two or more falls $[5,7,10,12,13]$, in a 3 - to 12 -month followup period.

The consequences of falls are numerous and can affect not only fallers, but also their family and community. Falls can result in injuries such as head trauma and hip fractures and may also contribute to increased fear of falling, reduced level of activity, and functional impairment, leading to decreased quality of life in these individuals [14-16]. The financial cost of a fall is high, for patients and the health system, as the fallrelated injuries, especially the fractures, are a common reason of hospitalization [17].

Several risk factors of falls have been proposed, although we can note that some of them have limited use in the general clinical practice, such as longer disease duration and dementia [8]; abnormal posture, freezing of gait (FOG), and frontal impairment [9]; and female gender, older age, and symmetrical onset [18]. Previous authors have found poor balance confidence and longer Timed Up and Go Test (TUG) as factors related with falls [19], reflecting the role of balance-related measures in fall risk assessment. Some functional activities have been described as common situations of falls in people with PD, such as transfers, bending or reaching for an object, turning, and walking [14], and these tasks are addressed by some balance scales [20-25]. 
In a meta-analysis of prospective studies of falling in $\mathrm{PD}$, the best predictor of falls was the presence of two or more falls in the previous year [26]. Although this is important for predicting future falls, this is not a modifiable risk factor and does not help to identify patients at risk before their first fall.

Considering the wide range and scope of falls consequences, it is of paramount importance to increase our knowledge of factors associated with recurrent falls, in particular, those identifiable through common clinical measures. This will provide useful information for identification of individuals at risk of falling and may contribute to the development of specific interventions to prevent falls.

The goals of this study were to report the frequency of falls in a sample of community-dwelling individuals with PD and to identify risk factors for recurrent falls, testing simultaneously the ability of disease-specific and balancerelated measures to predict falls.

\section{Methods}

2.1. Participants. The study sample consisted of consecutive individuals with PD with independent walking ability who attended the Movement Disorders Clinic at the State of Bahia Centre for the Elderly Health Attention, Brazil. Independent walking ability was defined when the patient was able to walk without assistance of another person, with or without a walking aid. The movement disorders clinic is a reference service for the state of Bahia, where approximately 500 elderly ( $\geq 60$ years old) patients with PD and other parkinsonian syndromes are followed, mostly from the public health system. The diagnosis of idiopathic PD was made by a certified neurologist, in accordance with the UK Parkinson's Disease Society Brain Bank Diagnostic Criteria [27]. Exclusion criteria were other neurological disorders than $\mathrm{PD}$, cognitive impairment (assessed by the mini-mental state examination and based on determined cutoff scores by previous authors, in accordance with the level of education of each participant) [28], severe visual disturbance, vestibular dysfunction, and musculoskeletal problems that might affect balance.

Patients were recruited between April of 2010 and January of 2012. The study was approved by the local research ethics committee. Initially, potential participants were approached by a physical therapist (L.R.S.A) and explanations related to the study were given to them. After that, each individual who decided to participate in the study provided written informed consent.

2.2. Assessments. Demographic and clinical data were recorded including the following information: age, gender, disease duration, history of falls, antiparkinsonian medications taken, presence of motor fluctuations, dyskinesia, FOG, and comorbidities (cardiovascular diseases, such as high blood pressure, heart failure, arrhythmia, hypercholesterolemia, peripheral vascular diseases, and diabetes; depression; urinary incontinence; constipation; osteoporosis). The definition of each comorbidity was based on self-report complemented by chart review. The levodopa equivalent dose (LED) was calculated based on a previously reported conversion factor [29]. A fall was defined as "an event which results in a person coming to rest unintentionally on the ground or other level, not as the result of a major intrinsic event or overwhelming hazard" [30]. Subjects were classified as nonfallers if they reported none or one fall in the preceding 12 months and as recurrent fallers if they reported two or more falls in the preceding 12 months. This group division was performed due to evidence supporting differences on clinical features and fall characteristics between individuals with PD who had experienced a single fall and those who are recurrent fallers and similarities between individuals with PD with no falling history and only a single fall [11].

2.3. PD-Specific Scales. The UPDRS was completed, including the activities of daily living (ADL) and motor sections, to assess both motor disability and motor impairment. The ADL section ranges from 0 to 52 points (greater disability) and the motor section from 0 to 108 (greater impairment) [31]. The modified Hoehn and Yahr scale (H\&Y) was used to evaluate the stage of PD (stage 5 means wheelchair-bound or bedridden) [32], and the Schwab and England scale (S\&E) to measure capacity for daily living (100\% indicates a completely independent person) [33]. The eight-item Parkinson's Disease Questionnaire (PDQ-8) was used to measure quality of life and is scored 0 to 100 (worst) [34]. The UPDRS was administered by a neurologist (G.T.V.) or physical therapist (L.R.S.A.), previously trained for this assessment.

2.4. Balance-Related Measures. Fear of falling was evaluated with two scales: the Activities-specific Balance Confidence Scale (ABC), which measures self-perceived balance confidence for completing daily living tasks, ranging from 0 to $100 \%$ (full confidence) $[15,20]$, and the Falls Efficacy ScaleInternational (FES-I), which assess concern about falling while performing certain activities of daily living, ranging from 16 to 64 points (highest concern) [21].

Balance and gait were assessed with four tests: Berg Balance Scale (BBS) [22], Functional Reach Test (FRT) [23], Timed Up and Go Test (TUG) [24], and Dynamic Gait Index (DGI) [25]. The BBS is an assessment tool for static and dynamic balance, which consists of 14 tasks related to common movements of daily living, such as transfers, turning, and balancing on reduced base of support, and is scored 0 to 56 points (best) $[22,35]$. The FRT is used to measure anteroposterior stability while standing with a stable base of support, recorded in centimeters $[23,36]$. The TUG assesses balance and functional mobility, in which patients arise from an armchair, walk for 3 meters, turn, walk back to the chair, and sit down, with results recorded in seconds. Patients were allowed to wear their regular footwear and use their customary walking aid [24, 37]. The DGI quantifies dynamic balance during gait, including tasks such as walking forward, with head turns and changing gait speed, ranging from 0 to 24 points (best) $[25,38]$.

All patients were assessed in the movement disorder clinic on the same occasion as they were recruited. They were evaluated during the "on" phase of the medication cycle and the balance measures were administered in the order described above by a trained physical therapist (L.R.S.A.). 
Participants were allowed to rest as needed during the balance assessments. Only the TUG was performed twice, and the first trial was considered as practice [24]. All the measurements took approximately 60 minutes to be performed.

2.5. Statistical Analysis. Statistics were performed using SPSS for Windows (version 16.0). Descriptive statistics were calculated for demographic and clinical variables, while comparison of recurrent fallers and nonfallers was completed using the Mann-Whitney test for continuous variables and the Pearson's chi-square test for categorical variables. To identify collinearity between the functional capacity scales, the fear of falling measures, and the balance tests, the Spearman correlation was performed and a value of $r \geq 0.75$ was set. Rates of falling were reported with $95 \%$ confidence intervals (CI). A significance level of 0.05 was set for these statistical tests.

Variables with $P<0.1$ were selected to enter into the multivariate regression analysis. To improve the clinical utility of the disease-specific, fear of falling, and balance measures, receiver operating characteristic (ROC) curves were constructed to determine the cutoff scores that would be best related to recurrent fallers, based on Youden indices [39]. The area under the curve (AUC), sensitivity, and specificity were determined for each test, as well as the 95\% CI. These measures were entered into the multivariate regression model with the cutoff scores previously chosen, as dichotomous variables. A backward stepwise (likelihood ratio-based) logistic regression was carried out, with falls history as the dependent variable $(0=$ no falls or one fall; $1=$ two or more falls). A significance level of 0.05 was set for variables remaining in the model. The proportion of variance explained by the logistic regression model was estimated by Nagelkerke's pseudo- $R$-squared.

\section{Results}

During the recruitment period, a total of 515 people attended the movement disorders clinic and 171 individuals with PD were enrolled in the study. One hundred and ninety-one patients were excluded because they did not meet the inclusion criteria for the following reasons: atypical parkinsonism $(n=58)$, essential tremor $(n=73)$, dystonia $(n=56)$, spinocerebellar ataxia $(n=2)$, and chorea $(n=2)$. One hundred and two people with PD were excluded due to other neurological conditions in addition to idiopathic PD ( $n=$ $10)$, cognitive impairment $(n=32)$, musculoskeletal problems that affected balance $(n=15)$, severe visual disturbance $(n=3)$, and vestibular dysfunction $(n=5)$ and because they were unable to walk independently $(n=37)$. An additional 51 individuals with PD were approached, but 16 were not regularly taking antiparkinsonian medication and 35 were unwilling to consent to participate in the study. This number $(n=51)$ corresponds to $23 \%$ of all participants who met all the eligibility criteria $(n=222)$.

The final sample consisted of 171 patients. The median age was 70 years (range 60-94) and 89 (52\%) were female. Patients had a median H\&Y stage of 2.5 (range 1.0-4.0) and the median LED was $600 \mathrm{mg}$ (range 300-1621.9). Seventyfour $(43 \%, 95 \%$ CI $36 \%-51 \%)$ patients reported one or more falls in the previous 12 months. Most patients (30\%, 95\% CI $24 \%-38 \%)$ reported two or more falls and were classified as recurrent fallers.

3.1. Comparison between Recurrent Fallers and Nonfallers. Demographic and disease-specific characteristics of the sample are shown in Table 1. Recurrent fallers had longer disease duration and greater disease severity according to $\mathrm{H} \& \mathrm{Y}$ stage, UPDRS motor score, and LED. They had functional limitations based on the UPDRS ADL and S\&E scale, and lower scores on the PDQ-8. Recurrent fallers also had worse performance on the BBS, DGI, FRT, and TUG, as well as higher degree of fear of falling based on FES-I and ABC $(P<0.001)$, as shown in Table 1 . Dyskinesia $(P=0.001)$, FOG $(P<0.001)$, urinary incontinence $(P<0.001)$, and constipation $(P=0.002)$ were associated with recurrent falls. Motor fluctuations were not associated with recurrent falls, but we found a borderline $P$ value of 0.084 .

3.2. ROC Analysis. The chosen cutoff scores and validity indices were as follows for the disease-specific measures: $\mathrm{H} \& \mathrm{Y}>2.5$ (AUC $=0.77,95 \%$ CI 0.70-0.83; sensitivity = $0.67,95 \%$ CI $0.53-0.80$; specificity $=0.76,95 \%$ CI $0.67-0.83$ ); UPDRS-motor $>33$ points $($ AUC $=0.78,95 \%$ CI $0.71-0.84$; sensitivity $=0.80,95 \%$ CI $0.66-0.90$; specificity $=0.72,95 \%$ CI $0.63-0.80)$; S\&E $\leq 70 \%$ (AUC $=0.77,95 \%$ CI $0.70-0.83$; sensitivity $=0.58,95 \%$ CI $0.43-0.71$; specificity $=0.86,95 \%$ CI 0.79-0.92); and UPDRS-ADL $>16$ points (AUC $=0.86,95 \%$ CI $0.80-0.91$; sensitivity $=0.67,95 \%$ CI $0.53-0.80$; specificity $=0.92,95 \%$ CI $0.85-0.96$ ).

In relation to the fear of falling scales, the cutoff scores and the corresponding values were FES-I $>30$ points (AUC $=0.81,95 \%$ CI 0.74-0.87; sensitivity $=0.88,95 \%$ CI 0.76-0.96; specificity $=0.66,95 \%$ CI $0.57-0.75$ ) and $\mathrm{ABC} \leq 46 \%$ (AUC $=0.83$, 95\% CI 0.76-0.88; sensitivity $=0.71,95 \%$ CI 0.57-0.83; specificity $=0.79,95 \%$ CI $0.71-0.86)$.

Regarding the balance measures, the determined cutoff scores and validity indices were $\mathrm{BBS} \leq 48$ points (AUC $=$ 0.86, 95\% CI 0.80-0.91; sensitivity $=0.81,95 \%$ CI $0.67-0.90$; specificity $=0.73,95 \%$ CI $0.64-0.81) ; \mathrm{DGI} \leq 18$ points $(\mathrm{AUC}=$ $0.84,95 \%$ CI $0.77-0.89$; sensitivity $=0.79,95 \%$ CI $0.65-0.89$; specificity $=0.74,95 \%$ CI $0.65-0.82)$; FRT $\leq 17$ centimeters $(\mathrm{AUC}=0.79,95 \%$ CI $0.72-0.85$; sensitivity $=0.75,95 \% \mathrm{CI}$ $0.61-0.86$; specificity $=0.76,95 \%$ CI $0.68-0.84$ ); TUG $>16.6$ seconds (AUC $=0.76,95 \%$ CI $0.69-0.82$; sensitivity $=0.69$, $95 \%$ CI $0.55-0.81$; specificity $=0.76,95 \%$ CI $0.68-0.84)$.

3.3. Multivariate Logistic Regression Model. In the multivariate analysis, 164 patients were included due to missing data related to the UPDRS motor score, which was entered into the model. The H\&Y score and the LED were also used as measures of disease severity rather than disease duration. Collinearity was found between the UPDRS-ADL and S\&E scale, and the first one was selected to enter into the model due to its higher AUC. To remove redundancy, FOG, also used as a dichotomous variable, was excluded from the model as it is an item from the UPDRS-ADL. As both the ABC and FES-I scales assess fear of falling during everyday tasks and they had similar values of AUC, we chose to enter the FES-I 
TABLE 1: Demographic, disease-specific characteristics and balance-related measures of the sample and comparison between recurrent fallers and nonfallers.

\begin{tabular}{|c|c|c|c|}
\hline \multirow{2}{*}{ Characteristics } & Recurrent fallers $(n=52)$ & Nonfallers $(n=119)$ & \multirow{2}{*}{$P$ value } \\
\hline & Median (min-max) & Median (min-max) & \\
\hline Age (years) & $71(61 ; 85)$ & $70(55 ; 94)$ & $0.355^{*}$ \\
\hline Disease duration (years) & $8.5(1 ; 31)$ & $4(1 ; 20)$ & $<0.001^{*}$ \\
\hline LED (mg) & $775(300 ; 1500)$ & $500(300 ; 1621.9)$ & $<0.001^{*}$ \\
\hline Modified Hoehn and Yahr & $3(2 ; 4)$ & $2.5(1 ; 4)$ & $<0.001^{*}$ \\
\hline UPDRS ADL & $19(2 ; 29)$ & $9(0 ; 24)$ & $<0.001^{*}$ \\
\hline UPDRS Motor & $43(13 ; 65)$ & $25.5(7 ; 59)$ & $<0.001^{*}$ \\
\hline Schwab and England (\%) & $70(40 ; 100)$ & $90(40 ; 100)$ & $<0.001^{*}$ \\
\hline PDQ-8 & $42.2(0 ; 93.7)$ & $25(0 ; 78.1)$ & $<0.001^{*}$ \\
\hline FES-I & $40(23 ; 62)$ & $27(16 ; 59)$ & $<0.001^{*}$ \\
\hline $\mathrm{ABC}(\%)$ & $37.2(1.9 ; 81.9)$ & $63.1(16.2 ; 100)$ & $<0.001^{*}$ \\
\hline BBS & $42(27 ; 54)$ & $52(37 ; 56)$ & $<0.001^{*}$ \\
\hline DGI & $16(8 ; 22)$ & $21(10 ; 24)$ & $<0.001^{*}$ \\
\hline FRT (centimeters) & $14.5(7 ; 32)$ & $20.5(6 ; 34)$ & $<0.001^{*}$ \\
\hline TUG (seconds) & $20.2(7.9 ; 149.5)$ & $14.2(7.0 ; 36.4)$ & $<0.001^{*}$ \\
\hline
\end{tabular}

${ }^{*}$ Mann-Whitney test.

LED: levodopa equivalent dose; UPDRS: Unified Parkinson's Disease Rating Scale; ADL: activities of daily living; PDQ-8: eight-item Parkinson's Disease Questionnaire; FES-I: Falls Efficacy Scale-International; ABC: Activities-specific Balance Confidence Scale; BBS: Berg Balance Scale; DGI: Dynamic Gait Index; TUG: Timed Up and Go Test; FRT: Functional Reach Test.

TABLE 2: Multivariate logistic regression analysis of the sample $(n=164)$.

\begin{tabular}{lcc}
\hline Variables & Odds ratio $(95 \%$ CI $)$ & $P$ value \\
\hline LED (per 100 mg increase) & $1.283(1.092-1.507)$ & 0.002 \\
BBS $\leq 48$ points & $3.868(1.175-12.738)$ & 0.026 \\
FES-I $>30$ points & $5.956(1.57-22.598)$ & 0.009 \\
UPDRS-ADL $>16$ points & $10.042(3.568-28.264)$ & $<0.001$ \\
\hline
\end{tabular}

CI: confidence interval; LED: levodopa equivalent dose; UPDRS-ADL: Unified Parkinson's Disease Rating Scale-activities of daily living; FES-I: Falls Efficacy Scale-International; BBS: Berg Balance Scale.

into the model, due to its higher value of sensitivity. As we found collinearity between all balance measures, the BBS was entered into the model due to its higher AUC.

The independent risk factors for recurrent falls in the multivariate model were LED, UPDRS-ADL > 16 points, FESI $>30$ points, and $\mathrm{BBS} \leq 48$ points (Table 2 ). The final model explained $64.6 \%$ of the variance of the history of recurrent falls.

\section{Discussion}

This study aimed to report the frequency of falls in a sample of individuals with PD and to identify risk factors for recurrent falls. The reported frequency of one or more falls was $43 \%$ and of two or more falls $30 \%$. These findings confirm that falling is a common feature of $\mathrm{PD}$. When analyzing retrospective studies, our reported frequency of one or more falls was lower than that found by Landers et al. (51\%) [40] and Dibble and Lange (55\%) [41], but the frequency of recurrent falls was similar to that obtained by Leddy et al. (31\%) [42]. As expected, our rate of falling was lower than that found by the majority of prospective studies (45-68\%) [5-10, 12]. However, it is of interest to note that, regarding recurrent falls, our rate was similar to that obtained by Duncan et al. (32\%) [13], lower than those reported by Gray and Hildebrand (41\%) [5] and Cole et al. (43\%) [12], but higher than that related by Kerr et al. (24\%) [10] and Bloem et al. (25\%) [7]. These differences may be due to different follow-up periods and characteristics of the sample in terms of disease severity.

Our main results were the independent association between recurrent fallers and higher LED and the association between recurrent fallers and three clinical scales (BBS, FESI, and UPDRS-ADL). Recurrent fallers had longer disease duration and increased disease severity based on the H\&Y and UPDRS motor sections than nonfallers, as well as higher LED. Recurrent fallers also showed reduced quality of life, higher degree of fear of falling and functional impairment, and decreased performance on all balance measures. These results are in agreement with those reported in previous studies $[10,11,40,41]$. Together, these results suggest that the LED (corresponding with disease severity), BBS $\leq 48$ points (showing postural instability), FES-I $>30$ points (representing fear of falling), and UPDRS-ADL > 16 points (indicating functional impairment) could help identify patients at a higher risk of falling as those with this profile seem to be at risk for becoming or being recurrent fallers. 
Previous research found that H\&Y stage, UPDRS motor score, and disease duration were considered risk factors for falls in multivariate analyses [7, 8, 10, 43, 44]. In this study, the LED was a factor related to falls. Kerr et al. [10] found that levodopa medications were slightly correlated with postural sway on the firm surface when all patients were considered, but there were no correlations when analyzing fallers and nonfallers separately. Previous studies have reported that postural instability is not sufficiently responsive to dopamine replacement due to the involvement of nondopaminergic pathways in gait and balance dysfunction [4, 45, 46], which can explain the higher level of balance impairment in individuals with PD who are recurrent fallers, even with higher levodopa dosages. The LED remained an independent risk factor for falling, probably because of its relation with longer PD duration, which is typically characterized by greater disease severity and postural instability, characteristics significantly higher in recurrent fallers.

The UPDRS-ADL > 16 points was also found to be an independent risk factor for recurrent falls. This subscale comprises some activities that can be difficult to perform not only because of motor limitations, but also due to postural instability, such as dressing and hygiene. Moreover, it rates walking difficulty and occurrence of FOG, factors linked to balance impairment, in addition to frequency of falling. FOG is a disabling feature of PD that occurs more often with disease progression and is related to falls in these patients $[5,47]$. The presence of FOG as well as scores on the FOG questionnaire has been considered independent related to falls in multivariate models [9, 10, 43]. Matinolli et al. [48] in a two-year prospective study about falls and mortality in PD also described the UPDRS-ADL as an independent risk factor for falling. Kerr et al. [10] in a six-month prospective study about predictors of falls in PD suggested a multivariate model including the UPDRS total score but reported that it can be substituted by the UPDRS-ADL, as they obtained similar sensitivity and specificity when this score was used. There are reliable formulas for transforming scores from the UPDRSADL to the recent developed MDS-UPDRS scale [49].

The BBS, with a cutoff score of $\leq 48$ points, remained an independent risk factor for falling. The BBS is a wellestablished tool to assess postural instability in people with PD [50-52] and previous studies have shown its greatest overall ability for predicting falls when compared with the other scales used in this study [40, 41]. Previous authors used different tools to evaluate postural instability, such as the Romberg test, Tinetti index, retropulsion test, and measurements of postural sway [7-10,53], which can explain the diversity of results about the clinical validity of balance tests to predict falls in individuals with PD. The Tinetti total score remained a predictor of falls in the multivariate model suggested by Kerr et al. [10] and its balance subscale was independently associated with falls in Contreras and Grandas' Study [44]. Lim et al. [43] and Mak and Pang [19] proposed a model including the TUG as an independent risk factor for falls, but it was the only balance test included in their study.

Recurrent fallers had higher degree of fear of falling on both $\mathrm{ABC}$ and FES-I. ABC scores have been determined to be independently associated with increased risk of falling in individuals with PD $[19,54]$. In accordance with these findings, we showed that a FES-I $>30$ points, reflecting fear of falling, was an independent risk factor for falling. It has been shown that fear of falling correlates with performance-based balance and mobility tests, as well as with functional capacity and disease severity [16], factors related to falls. It is important to note that this fear of falling may be protective if it increases caution during performance in daily activities, but it can be a negative factor when it leads to restrictions of mobility and social isolation, contributing to functional decline and to an actual increase in the risk of falling $[16,19]$.

Other variables were associated with recurrent falls, although they were not found to be independent risk factors in the presence of the other factors included in our analysis. Recurrent fallers presented more dyskinesia than nonfallers. This high frequency of dyskinesia could be explained by the higher intake of levodopa showed by recurrent fallers [1]. It has been shown that dyskinesia may contribute to postural instability in advanced PD [55] and previous authors also found that dyskinesia was associated with falls $[10,53]$. We believe that the presence of dyskinesia could contribute to an increased postural sway, especially when the trunk and feet are affected.

Urinary incontinence and constipation, both considered signs of autonomic dysfunction in PD, were associated with falls. Although we have not investigated thoroughly urinary incontinence, it is known that nocturia and urinary urgency are common events in PD [1]. Usually during the night, individuals with PD are without the effect of levodopa, which may generate greater motor difficulties and risk of falling when standing and walking to the toilet. Balash et al. [56] in a study about falls with 350 patients with PD identified urinary incontinence as an independent risk factor for being a recurrent faller. The association between constipation and falls is discussed in clinical practice probably due to its contribution to decreased absorption of levodopa [57].

Motor fluctuations might be associated with postural instability and higher risk of falling, as it generates reduction of motor performance [58], leading to functional impairment and gait difficulty. Some authors have found association between falls and motor fluctuations [8, 44], whereas others have reported only borderline $P$ value [53], similar to our findings.

The main strength of our study was to simultaneously test disease-specific and balance-related measures in the same sample population, suggesting the importance of using common clinical scales to assess risk of falling. The utilization of the scales that we found to be associated with recurrent falls, with their respective cutoff scores, may be useful in clinical practice. These scales provide detailed information about impaired functional activities and balance-related tasks and can be used not only in the assessment of the individuals with $\mathrm{PD}$, but also during treatment, and to evaluate treatment outcomes. Our results also provide evidence for an independent validation sample using these scales, selecting the best combination of measures to determine the risk of recurrent falls. Although our score performed well in our 
patient sample, an independent validation sample is important before incorporation into clinical practice.

Differences found in this study may be due to different methods applied by previous authors to identify risk factors associated with falls, different classification of fallers and nonfallers, and different sample characteristics. One limitation of this study is the retrospective classification of recurrent fallers and nonfallers. However, our rate of recurrent fallers was similar to that reported by previous prospective studies. We are currently carrying out a one-year prospective study and further analysis may differentiate predictors of single and recurrent falls.

\section{Conclusions}

The occurrence of falls in $\mathrm{PD}$ is high and recurrent falls, especially, can play an important role in the functional status of patients. The identification of risk factors associated with recurrent falls in people with $\mathrm{PD}$, especially those that are modifiable, could be useful to target intervention programs to improve balance and possibly reduce the risk of falls and related injuries. We encourage the use of the UPDRS-ADL section, BBS, and FES-I in screening for falls risk in individuals with PD, measures that could be easily performed either in a home or outpatient clinical setting.

\section{Conflict of Interests}

The authors declare that there is no conflict of interests regarding the publication of this paper.

\section{Acknowledgments}

The authors thank Antônio Cunha Porto Maia for his statistical analysis and consultations and Rebecca A. Gruber for her review of the paper.

\section{References}

[1] C. W. Olanow, M. B. Stern, and K. Sethi, "The scientific and clinical basis for the treatment of Parkinson disease (2009)," Neurology, vol. 72, no. 21, pp. S1-S136, 2009.

[2] F. B. Horak, D. Dimitrova, and J. G. Nutt, "Direction-specific postural instability in subjects with Parkinson's disease," Experimental Neurology, vol. 193, no. 2, pp. 504-521, 2005.

[3] T. A. Boonstra, H. Van Der Kooij, M. Munneke, and B. R. Bloem, "Gait disorders and balance disturbances in Parkinson's disease: clinical update and pathophysiology," Current Opinion in Neurology, vol. 21, no. 4, pp. 461-471, 2008.

[4] Y. A. M. Grimbergen, M. Munneke, and B. R. Bloem, "Falls in Parkinson's disease," Current Opinion in Neurology, vol. 17, no. 4, pp. 405-415, 2004.

[5] P. Gray and K. Hildebrand, "Fall risk factors in Parkinson's disease," Journal of Neuroscience Nursing, vol. 32, no. 4, pp. 222228, 2000.

[6] A. Ashburn, E. Stack, R. M. Pickering, and C. D. Ward, "Predicting fallers in a community-based sample of people with Parkinson's disease," Gerontology, vol. 47, no. 5, pp. 277-281, 2001.
[7] B. R. Bloem, Y. A. M. Grimbergen, M. Cramer, M. Willemsen, and A. H. Zwinderman, "Prospective assessment of falls in Parkinson's disease," Journal of Neurology, vol. 248, no. 11, pp. 950-958, 2001.

[8] B. H. Wood, J. A. Bilclough, A. Bowron, and R. W. Walker, "Incidence and prediction of falls in Parkinson's disease: a prospective multidisciplinary study," Journal of Neurology Neurosurgery and Psychiatry, vol. 72, no. 6, pp. 721-725, 2002.

[9] M. D. Latt, S. R. Lord, J. G. L. Morris, and V. S. C. Fung, "Clinical and physiological assessments for elucidating falls risk in Parkinson's disease," Movement Disorders, vol. 24, no. 9, pp. 1280-1289, 2009.

[10] G. K. Kerr, C. J. Worringham, M. H. Cole, P. F. Lacherez, J. M. Wood, and P. A. Silburn, "Predictors of future falls in Parkinson disease," Neurology, vol. 75, no. 2, pp. 116-124, 2010.

[11] M. K. Y. Mak and M. Y. C. Pang, "Parkinsonian single fallers versus recurrent fallers: different fall characteristics and clinical features," Journal of Neurology, vol. 257, no. 9, pp. 1543-1551, 2010.

[12] M. H. Cole, P. A. Silburn, J. M. Wood, C. J. Worringham, and G. K. Kerr, "Falls in Parkinson's disease: kinematic evidence for impaired head and trunk control," Movement Disorders, vol. 25, no. 14, pp. 2369-2378, 2010.

[13] R. P. Duncan, A. L. Leddy, J. T. Cavanaugh et al., "Accuracy of fall prediction in parkinson disease: six-month and 12-month prospective analyses," Parkinson's Disease, vol. 2012, Article ID 237673, 7 pages, 2012.

[14] A. Ashburn, E. Stack, C. Ballinger, L. Fazakarley, and C. Fitton, "The circumstances of falls among people with Parkinson's disease and the use of Falls Diaries to facilitate reporting," Disability and Rehabilitation, vol. 30, no. 16, pp. 1205-1212, 2008.

[15] A. L. Adkin, J. S. Frank, and M. S. Jog, "Fear of falling and postural control in Parkinson's disease," Movement Disorders, vol. 18, no. 5, pp. 496-502, 2003.

[16] F. Franchignoni, E. Martignoni, G. Ferriero, and C. Pasetti, "Balance and fear of falling in Parkinson's disease," Parkinsonism and Related Disorders, vol. 11, no. 7, pp. 427-433, 2005.

[17] J. J. Watts, J. L. McGinley, F. Huxham et al., "Cost effectiveness of preventing falls and improving mobility in people with Parkinson disease: protocol for an economic evaluation alongside a clinical trial," BMC Geriatrics, vol. 8, article 23, 2008.

[18] D. R. Williams, H. C. Watt, and A. J. Lees, "Predictors of falls and fractures in bradykinetic rigid syndromes: a retrospective study," Journal of Neurology, Neurosurgery \& Psychiatry, vol. 77, no. 4, pp. 468-473, 2006.

[19] M. K. Y. Mak and M. Y. C. Pang, "Balance confidence and functional mobility are independently associated with falls in people with Parkinson's disease," Journal of Neurology, vol. 256, no. 5, pp. 742-749, 2009.

[20] L. E. Powell and A. M. Myers, "The activities-specific balance confidence (ABC) scale," Journals of Gerontology. Series A Biological Sciences and Medical Sciences, vol. 50, no. 1, pp. M28M34, 1995.

[21] F. F. O. Camargos, R. C. Dias, J. M. D. Dias, and M. T. F. Freire, "Cross-cultural adaptation and evaluation of the psychometric properties of the Falls Efficacy Scale - International Among Elderly Brazilians (FES-I-BRAZIL)," Revista Brasileira de Fisioterapia, vol. 14, no. 3, pp. 237-243, 2010.

[22] K. O. Berg, S. L. Wood-Dauphinée, J. I. Williams, and B. Maki, "Measuring balance in the elderly: validation of an instrument," Canadian Journal of Public Health, vol. 83, supplement 2, pp. S7-S11, 1992. 
[23] P. W. Duncan, S. Studenski, J. Chandler, and B. Prescott, "Functional reach: predictive validity in a sample of elderly male veterans," Journals of Gerontology, vol. 47, no. 3, pp. M93-M98, 1992.

[24] A. Shumway-Cook, S. Brauer, and M. Woollacott, "Predicting the probability for falls in community-dwelling older adults using the timed up \& go test," Physical Therapy, vol. 80, no. 9, pp. 896-903, 2000.

[25] A. Shumway-Cook and M. H. Woolacott, "Control of posture and balance," in Motor Control Theory and Practical Applications, A. Shumway-Cook and M. H. Woolacott, Eds., p. 120, Williams \& Wilkins, Baltimore, Md, USA, 1995.

[26] R. M. Pickering, Y. A. M. Grimbergen, U. Rigney et al., "A metaanalysis of six prospective studies of falling in Parkinson's disease," Movement Disorders, vol. 22, no. 13, pp. 1892-1900, 2007.

[27] A. J. Hughes, S. E. Daniel, L. Kilford, and A. J. Lees, "Accuracy of clinical diagnosis of idiopathic Parkinson's disease: a clinicopathological study of 100 cases," Journal of Neurology Neurosurgery and Psychiatry, vol. 55, no. 3, pp. 181-184, 1992.

[28] P. H. F. Bertolucci, S. M. Brucki, S. R. Campacci, and Y. Juliano, "O mini-exame do estado mental em uma população: impacto da escolaridade," Arquivos de Neuro-Psiquiatria, vol. 52, no. 1, pp. 1-7, 1994.

[29] C. L. Tomlinson, R. Stowe, S. Patel, C. Rick, R. Gray, and C. E. Clarke, "Systematic review of levodopa dose equivalency reporting in Parkinson's disease," Movement Disorders, vol. 25, no. 15, pp. 2649-2653, 2010.

[30] R. D. Clark, S. R. Lord, and I. W. Webster, "Clinical parameters associated with falls in an elderly population," Gerontology, vol. 39, no. 2, pp. 117-123, 1993.

[31] S. Fahn, R. L. Elton, and Members of the UPDRS Committee, "Unified Parkinson's disease rating scale," in Recent Developments in Parkinson's Disease, S. Fahn, C. D. Marsden, M. Goldstein, and D. B. Calne, Eds., pp. 153-163, McMillan Health Care, New Jersey, NJ, USA, 1987.

[32] Y. J. Zhao, H. L. Wee, Y.-H. Chan et al., "Progression of Parkinson's disease as evaluated by Hoehn and Yahr stage transition times," Movement Disorders, vol. 25, no. 6, pp. 710-716, 2010.

[33] C. Ramaker, J. Marinus, A. M. Stiggelbout, and B. J. van Hilten, "Systematic evaluation of rating scales for impairment and disability in Parkinson's disease," Movement Disorders, vol. 17, no. 5, pp. 867-876, 2002.

[34] P. Martinez-Martin, M. Jeukens-Visser, K. E. Lyons et al., "Health-related quality-of-life scales in Parkinson's disease: critique and recommendations," Movement Disorders, vol. 26, no. 13, pp. 2371-2380, 2011.

[35] P. L. Scalzo, I. C. Nova, M. R. Perracini et al., "Validation of the Brazilian version of the berg balance scale for patients with Parkinson's disease," Arquivos de Neuro-Psiquiatria, vol. 67, no. 3 B, pp. 831-835, 2009.

[36] A. L. Behrman, K. E. Light, S. M. Flynn, and M. T. Thigpen, "Is the functional reach test useful for identifying falls risk among individuals with Parkinson's disease?" Archives of Physical Medicine and Rehabilitation, vol. 83, no. 4, pp. 538-542, 2002.

[37] S. Morris, M. E. Morris, and R. Iansek, "Reliability of measurements obtained with the timed "Up \& Go" test in people with Parkinson disease," Physical Therapy, vol. 81, no. 2, pp. 810-818, 2001.
[38] S. M. de Castro, M. R. Perracini, and F. F. Ganança, "Versão brasileira do dynamic gait index," Revista Brasileira de Otorrinolaringologia, vol. 72, no. 6, pp. 817-825, 2006.

[39] W. J. Youden, "Index for rating diagnostic tests," Cancer, vol. 3, no. 1, pp. 32-35, 1950.

[40] M. R. Landers, A. Backlund, J. Davenport, J. Fortune, S. Schuerman, and P. Altenburger, "Postural instability in idiopathic parkinson's disease: discriminating fallers from nonfallers based on standardized clinical measures," Journal of Neurologic Physical Therapy, vol. 32, no. 2, pp. 56-61, 2008.

[41] L. E. Dibble and M. Lange, "Predicting falls in individuals with Parkinson disease: a reconsideration of clinical balance measures," Journal of Neurologic Physical Therapy, vol. 30, no. 2, pp. 60-67, 2006.

[42] A. L. Leddy, B. E. Crowner, and G. M. Earhart, "Functional gait assessment and balance evaluation system test: reliability, validity, sensitivity, and specificity for identifying individuals with parkinson disease who fall," Physical Therapy, vol. 91, no. 1, pp. 102-113, 2011.

[43] I. Lim, E. van Wegen, D. Jones et al., "Identifying fallers with Parkinson's disease using home-based tests: who is at risk?" Movement Disorders, vol. 23, no. 16, pp. 2411-2415, 2008.

[44] A. Contreras and F. Grandas, "Risk of falls in Parkinson's Disease: a cross-sectional study of 160 patients," Parkinson's Disease, vol. 2012, Article ID 362572, 10 pages, 2012.

[45] B. R. Bloem, D. J. Beckley, J. Gert van Dijk, A. H. Zwinderman, M. P. Remler, and R. A. C. Roos, "Influence of dopaminergic medication on automatic postural responses and balance impairment in Parkinson's disease," Movement Disorders, vol. 11, no. 5, pp. 509-521, 1996.

[46] K. Sethi, "Levodopa unresponsive symptoms in Parkinson disease," Movement Disorders, vol. 23, no. S3, pp. S521-S533, 2008.

[47] B. R. Bloem, J. M. Hausdorff, J. E. Visser, and N. Giladi, "Falls and freezing of Gait in Parkinson's disease: a review of two interconnected, episodic phenomena," Movement Disorders, vol. 19, no. 8, pp. 871-884, 2004.

[48] M. Matinolli, J. T. Korpelainen, K. A. Sotaniemi, V. V. Myllylä, and R. Korpelainen, "Recurrent falls and mortality in Parkinson's disease: a prospective two-year follow-up study," Acta Neurologica Scandinavica, vol. 123, no. 3, pp. 193-200, 2011.

[49] C. G. Goetz, G. T. Stebbins, and B. C. Tilley, "Calibration of unified Parkinson's disease rating scale scores to Movement Disorder Society-unified Parkinson's disease rating scale scores," Movement Disorders, vol. 27, no. 10, pp. 1239-1242, 2012.

[50] A. A. Qutubuddin, P. O. Pegg, D. X. Cifu, R. Brown, S. McNamee, and W. Carne, "Validating the Berg Balance Scale for patients with Parkinson's disease: a key to rehabilitation evaluation," Archives of Physical Medicine and Rehabilitation, vol. 86, no. 4, pp. 789-792, 2005.

[51] K. J. Brusse, S. Zimdars, K. R. Zalewski, and T. M. Steffen, "Testing functional performance in people with Parkinson disease," Physical Therapy, vol. 85, no. 2, pp. 134-141, 2005.

[52] H. Tanji, A. L. Gruber-Baldini, K. E. Anderson et al., "A comparative study of physical performance measures in Parkinson's disease," Movement Disorders, vol. 23, no. 13, pp. 1897-1905, 2008.

[53] M. Matinolli, J. T. Korpelainen, R. Korpelainen, K. A. Sotaniemi, M. Virranniemi, and V. V. Myllylä, "Postural sway and falls in Parkinson's disease: a regression approach," Movement Disorders, vol. 22, no. 13, pp. 1927-1935, 2007. 
[54] M. K. Y. Mak and M. Y. C. Pang, "Fear of falling is independently associated with recurrent falls in patients with Parkinson's disease: a 1-year prospective study," Journal of Neurology, vol. 256, no. 10, pp. 1689-1695, 2009.

[55] S. Armand, T. Landis, R. Sztajzel, and P. R. Burkhard, "Dyskinesia-induced postural instability in Parkinson's disease," Parkinsonism \& Related Disorders, vol. 15, no. 5, pp. 359-364, 2009.

[56] Y. Balash, C. Peretz, G. Leibovich, T. Herman, J. M. Hausdorff, and N. Giladi, "Falls in outpatients with Parkinson's disease: frequency, impact and identifying factors," Journal of Neurology, vol. 252, no. 11, pp. 1310-1315, 2005.

[57] E. Ogawa, R. Sakakibara, M. Kishi, and F. Tateno, "Constipation triggered the malignant syndrome in Parkinson's disease," Neurological Sciences, vol. 33, no. 2, pp. 347-350, 2012.

[58] M. Stacy, "The wearing-off phenomenon and the use of questionnaires to facilitate its recognition in Parkinson's disease," Journal of Neural Transmission, vol. 117, no. 7, pp. 837-846, 2010. 


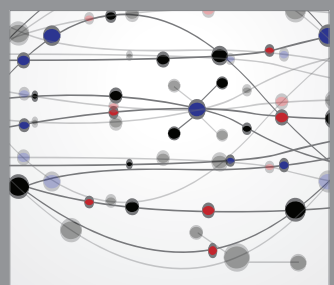

The Scientific World Journal
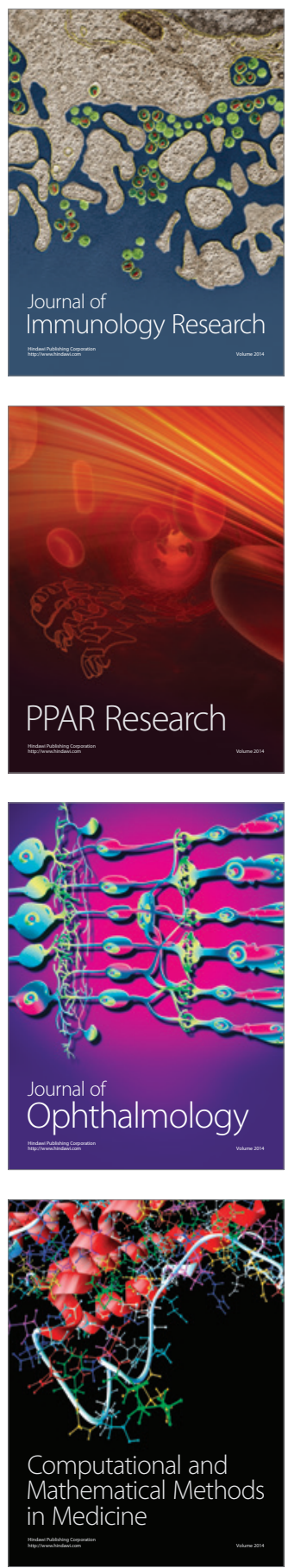

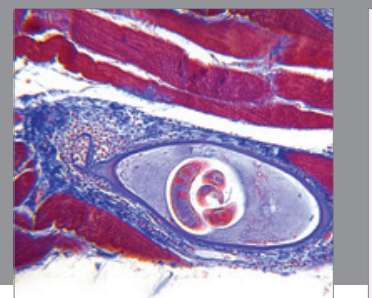

Gastroenterology

Research and Practice
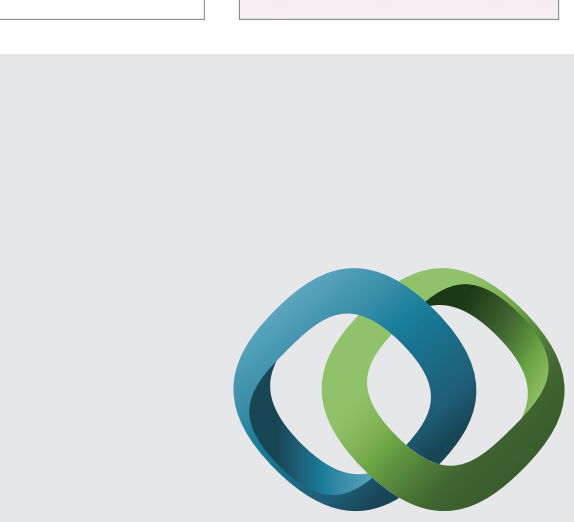

\section{Hindawi}

Submit your manuscripts at

http://www.hindawi.com
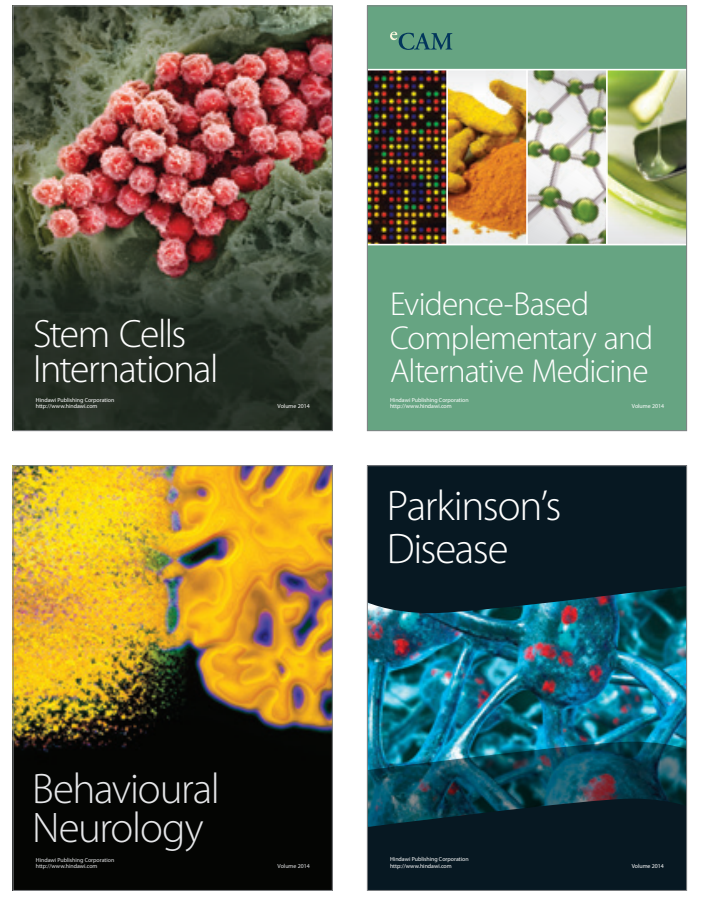
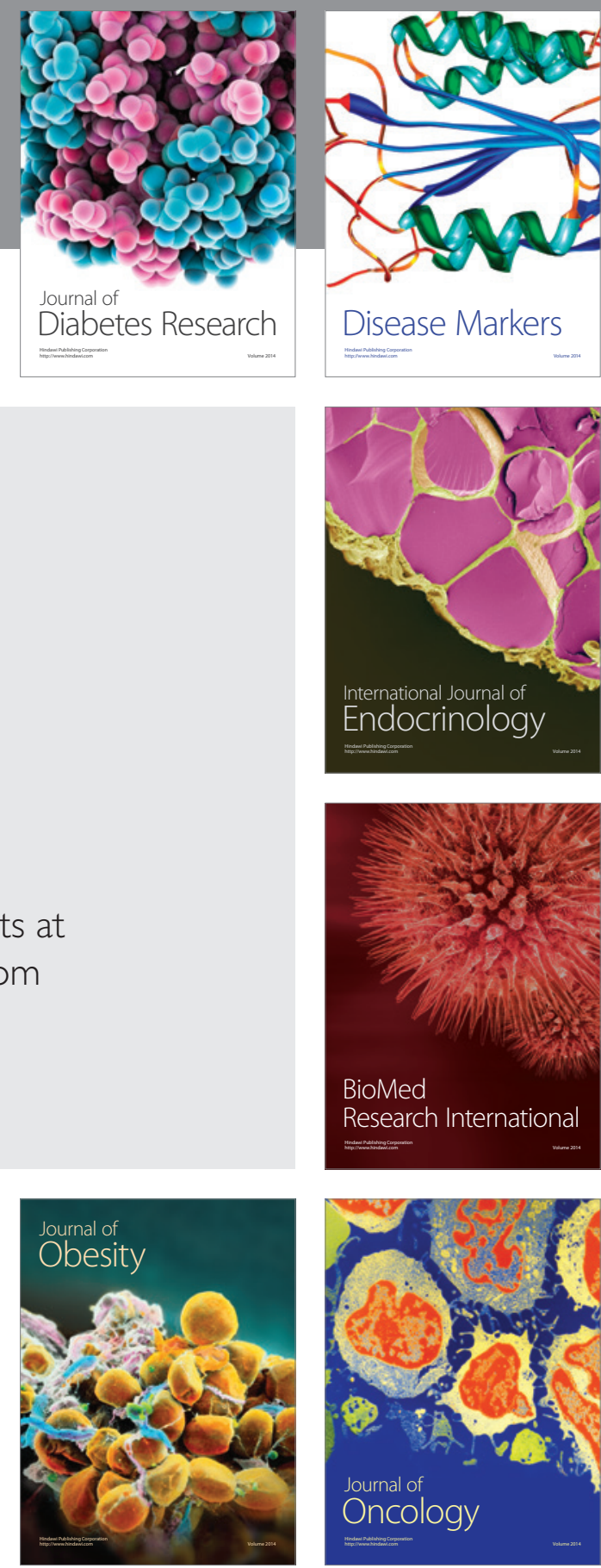

Disease Markers
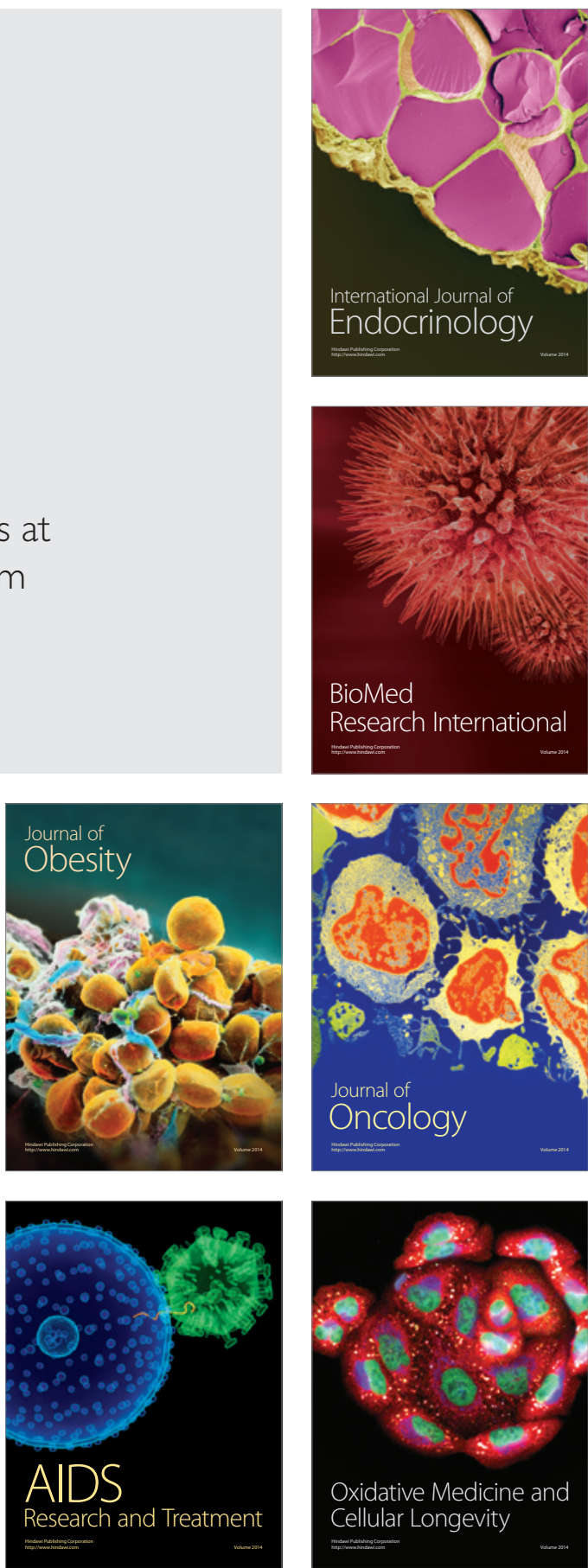